\title{
Disorders of fatty acid transport and mitochondrial oxidation: Challenges and dilemmas of metabolic evaluation
}

Piero Rinaldo, $M D, P h D$, and Dietrich Matern, $M D$

\begin{abstract}
Inborn errors of fatty acid transport and mitochondrial oxidation (FATMO) have drawn considerable attention in recent years for the rapid pace of discovery of new defects and an ever-increasing spectrum of clinical phenotypes. Several of these disorders are not detected by conventional biochemical investigations, even when a patient is symptomatic with fasting intolerance or functional failure of fatty acid dependent tissue(s). In our view, today's major challenges are the inclusion of FATMO disorders in newborn screening programs and the investigation of the role played by individual disorders in maternal complications of pregnancy, sudden and unexpected death in early life, and pediatric acute/fulminant liver failure. Dilemmas are found in the debate over the limitations, if any, to be imposed on the expansion of newborn screening using tandem mass spectrometry, in the provision of prenatal diagnosis for otherwise treatable disorders, and in the diagnostic workup of "unclassified" cases. Genetics in
\end{abstract} Medicine, 2000:2(6):338-344.

Key Words: fatty acid transport, mitochondrial oxidation, newborn screening, sudden death

Mitochondrial fatty acid oxidation plays a major role in energy production and homeostasis. ${ }^{1}$ In response to fasting, long-chain fatty acids are mobilized from adipose tissue and taken up by active transport in liver and muscle cells. Enzymes responsible for the $\beta$-oxidation of longer chain species are associated with the inner mitochondrial membrane, while the enzymes responsible for the metabolism of medium- and short-chain species are located within the mitochondrial matrix. A distinction between defects of membrane-bound and matrix enzymes has become increasingly relevant to the classification of inborn errors of this pathway because the number of defects in each group is almost equal, and the two groups tend to manifest with different patterns of clinical manifestations and particularly biochemical phenotypes. ${ }^{2}$

The pathogenetic mechanisms underlying the clinical manifestations of FATMO disorders are similar to virtually all mitochondrial inborn errors of metabolism, originating from either one of two basic mechanisms: intoxication and energy deficiency. ${ }^{3}$ In FATMO disorders, both mechanisms are involved (Fig. 1), and consequent signs and symptoms include hypoketotic hypoglycemia, transient to fulminant liver dis-

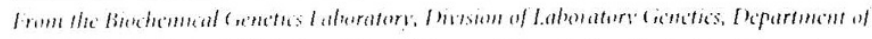

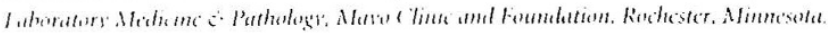

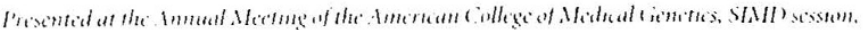

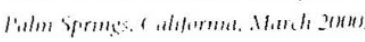

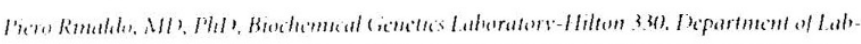

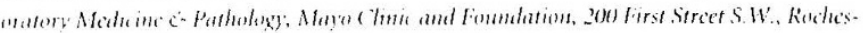
he. MIN 35015

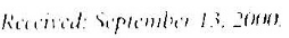

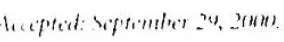

ease, skeletal myopathy, dilated/hypertrophic cardiomyopathy, and sudden and unexpected death in early life. ${ }^{2-4}$

Today's most pressing challenges are found first and foremost in the expansion of newborn screening programs to include FATMO disorders. Second, the challenge is to understand the role specific disorders may play in the etiology of maternal complications of pregnancy, sudden and unexpected death in early life, and acute/fulminant liver failure in childhood. Dilemmas, defined as difficult choices to be made between equally valid but contrasting solutions, are posed by the application of the full diagnostic potential of tandem mass spectrometry to newborn screening, the provision of prenatal diagnosis for treatable disorders, and by defining the boundaries of the diagnostic workup of "unclassified" cases.

\section{NEWBORN SCREENING OF FATMO DISORDERS: THE CHALLENGE}

State-mandated newborn screening was initiated in the early 1960 s for the identification of infants affected with phenylketonuria (PKU), but soon was expanded to include additional genetic and nongenetic conditions. The goal of newborn screening is the presymptomatic diagnosis of treatable disorders frequent enough to allow for a positive cost-benefit ratio. Tandem mass spectrometry (MS/MS) is a powerful multianalyte screening method which is ideally suited for populationwide testing. ${ }^{5}$ Since the early 1990 s, MS/MS has made possible the screening for several FATMO disorders based on the profiling of acylcarnitines in blood spots (Table 1). Beyond the availability of a screening method amenable to populationwide testing, compelling reasons for the inclusion of FATMO 


Intoxication
Fatty acids
Acylcarnitines
Ammonia, lactic acid
Uric acid
Energy deficiency
Hypoglycemia
†reducing equivalent to
OxPHOS
†ketone bodies to
extrahepatic tissues

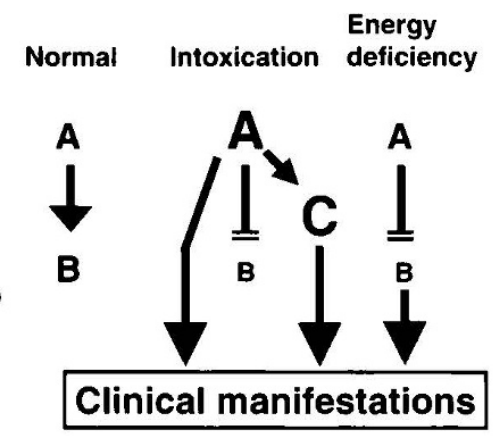

Fig. 1 Metabolic effects of impaired fatty acid metabolism. A and B indicate a substrate and product, respectively, of a generic enzyme reaction; $C$ indicates the product of an alternative pathway (for example, conjugation with carnitine and glycine). OXPHOS, oxidative phosphorylation.

disorders in screening programs are a documented prevention of morbidity by early diagnosis, and simple and inexpensive treatment strategies. This situation, however, has sparked an intense debate over the allocation of sufficient resources to provide treatment, follow-up care, and genetic counseling to a far more diverse population of patients.

To date, a consensus has been reached to screen without further delay at least for medium-chain acyl-CoA dehydrogenase (MCAD) deficiency and glutaric acidemia type $1 .{ }^{6} \mathrm{MCAD}$ deficiency is the most common fatty acid oxidation disorder and possibly one of the most frequent metabolic diseases overall; according to recent estimates, it occurs in approximately 1:6,500-1:17,000 live births. ${ }^{7}$ Although growth and development are not affected, the manifestations of acute episodes of decompensation include severe liver dysfunction, hypoglycemic coma, and sudden, unexpected death. The first acute episode usually occurs early in life, but affected individuals may present at any age, including adulthood. ${ }^{8}$ Sudden and unexpected death is the first manifestation of MCAD deficiency in $18 \%$ of patients, ${ }^{9}$ an outcome which could be prevented, with almost no exceptions, by simple dietary treatment and basic preventive measures.

\section{NEWBORN SCREENING OF FATMO DISORDERS: THE DILEMMA}

The reliability of screening by MS/MS for the majority of FATMO disorders, particularly long-chain L-3-hydroxy acylCoA dehydrogenase (LCHAD) deficiency, has not been proven conclusively based on the prospective detection of a significant number of affected cases (Table 1). Obviously, any questions about the specificity and sensitivity of a screening method lead to concerns of encountering high rates of falsenegative (missed diagnosis) and false-positive (unnecessary follow up) results, with a corollary of potential malpractice and liability issues. Despite some reassuring reports, ${ }^{10}$ the finding of only one case with LCHAD deficiency among almost one
Table 1

Tandem mass spectrometry in newborn screening of FATMO disorders

\begin{tabular}{|c|c|c|}
\hline Disorder & $\begin{array}{l}\text { Effectiveness } \\
\text { of early } \\
\text { treatment }{ }^{a}\end{array}$ & $\begin{array}{c}\text { Effectiveness } \\
\text { of } \\
\mathrm{MS} / \mathrm{MS}^{b}\end{array}$ \\
\hline \multicolumn{3}{|l|}{ Disorders of membrane-bound enzymes } \\
\hline Carnitine transport defect & +++ & $+1-$ \\
\hline Long-chain fatty acid transport defect & $+\$$ & - \\
\hline CPT-I deficiency (liver) & + & $(+)$ \\
\hline CACT deficiency & + & + \\
\hline \multicolumn{3}{|l|}{ CPT-II deficiency } \\
\hline Neonatal onset & - & + \\
\hline Late onset & + & + \\
\hline VLCAD deficiency & + & $(+)$ \\
\hline \multicolumn{3}{|l|}{ ETF-QO deficiency (GA2) } \\
\hline Neonatal onset & - & + \\
\hline Late onset & + & $(+)$ \\
\hline LCHAD deficiency & + & $(+)$ \\
\hline TFP deficiency $(\alpha, \beta)$ & $+\varsigma$ & $(+)$ \\
\hline \multicolumn{3}{|l|}{ Disorders of mitochondrial matrix enzymes } \\
\hline MCAD deficiency & +++ & + \\
\hline SCAD deficiency & + & + \\
\hline Functional SCAD deficiency & $?$ & + \\
\hline \multicolumn{3}{|l|}{ ETF deficiency ( $\alpha, \beta, \mathrm{GA} 2)$} \\
\hline Neonatal onset & - & + \\
\hline Late onset & + & $(+)$ \\
\hline Riboflavin responsive form(s) (GA2) & +++ & $(+)$ \\
\hline SCHAD deficiency (muscle) & + & - \\
\hline SCHAD deficiency (fibroblasts) & + & $+1-$ \\
\hline SCHAD deficiency (liver) & $+\S$ & - \\
\hline M/SCHAD deficiency & $+\$$ & $(+)$ \\
\hline MCKAT deficiency & + & $(+)$ \\
\hline 2,4-Dienoyl-CoA reductase deficiency & $?$ & $(+)$ \\
\hline HMG-CoA synthase deficiency & + & - \\
\hline HMG-CoA lyase deficiency & + & + \\
\hline
\end{tabular}

Abbreviations are as follows, in alphabetical order: CACT, carnitine acylcarnitine translocase; CPT, carnitine palmitoyltransferase; ETF, electron transfer flavoprotein; ETF-QO, electron transfer flavoprotein ubiquinone-oxidoreductase; GA2, glutaric acidemia type II; HMG, 3-hydroxy 3-methylglutaryl; LCHAD, long-chain L-3-hydroxy acyl-CoA dehydrogenase; MCAD, medium-chain acyl-CoA dehydrogenase; MCKAT, medium-chain 3-ketoacyl$\mathrm{CoA}$ thiolase; M/SCHAD, medium/short chain L-3-hydroxy acyl-CoA dehydrogenase; SCAD, short-chain acyl-CoA dehydrogenase; SCHAD, shortchain l-3-hydroxy acyl-CoA dehydrogenase; TFP, trifunctional protein; VLCAD, very long-chain acyl-CoA dehydrogenase.

"Effectiveness of treatment: +++ , demonstrated; + , dietary and preventive measures; -, no effective treatment; $\$$, liver transplantation; ?, insufficient information.

${ }^{b}$ Effectiveness of MS/MS: +, demonstrated in blood spots; $(+)$, expected to be effective, but not yet conclusively demonstrated; $+1-$, questionable effectiveness; - , not effective. 
million newborns screened by the laboratory with the largest experience in this field (Dr. Edwin W. Naylor, Neo Gen Screening; personal communication) appears to be in contrast with the number of new cases diagnosed after the onset of symptoms ${ }^{11}$ or post mortem. ${ }^{12}$ As there have been no reports of cases actually missed, LCHAD deficiency could be a disorder far less common than previously estimated. ${ }^{13}$

The robustness of MS/MS as a global screening method for FATMO disorders other than MCAD deficiency will be eventually proven only by an accelerated implementation and careful review of the outcome of prospective programs, a goal which could greatly benefit from active collaboration between multiple testing sites with proven analytical experience. These efforts should resolve another controversial issue, related to the effectiveness of MS/MS to detect disorders for which no effective treatment is available (for example, neonatal onset of glutaric acidemia type 2 and CPT-II deficiency). Although this is in contrast with conventional screening criteria, there is little doubt that an early diagnosis under these conditions is still beneficial, because it allows for proper genetic counseling, is provided at no added cost to the system, and could greatly contain the scope and cost of the diagnostic workup of an acutely ill newborn.

Two factors that have further complicated the ongoing debate over expanded newborn screening are first the mounting awareness in the general public, stirred by strong and vocal parent support groups, and second the special attention given by the media to this issue. On the other hand, coping with a drastically different analytical platform and the degree of complexity of result interpretation have contributed to the slow implementation of MS/MS by state-based screening laboratories. ${ }^{14}$ Of all possible outcomes of this volatile situation, the one that should most concern clinical and biochemical geneticists is the procrastinated and "spotted" addition of FATMO disorders to screening programs, practically creating a two-tier system (screened vs. not screened), which will be harshly criticized as most unfair to patients and their families.

\section{ROLE OF FATMO DISORDERS AS CAUSE OF MATERNAL COMPLICATIONS OF PREGNANCY}

A unique aspect of FATMO disorders is the associated risk of maternal complications during late pregnancy. Although this association has been best characterized for fetal LCHAD deficiency and acute fatty liver of pregnancy (AFLP), ${ }^{15}$ Table 2 shows that AFLP and other phenotypes have been reported in pregnant women carrying fetuses affected with at least five other FATMO disorders. ${ }^{16,17,17 a}$ Despite the possibly coincidental nature of isolated observations, ${ }^{18}$ a detailed inquiry of the past obstetric history should be a required component of the clinical evaluation of new patients with any FATMO disorder. Furthermore, routine monitoring of subsequent pregnancies in known families should include measures to achieve early detection of maternal complications.

In most inborn errors of metabolism, heterozygote mothers carrying affected fetuses provide a protective and compensatory effect to the metabolic block of the fetus, resulting in the delivery of healthy babies that will manifest signs of intoxication or energy deficiency only after a variable period of time. No unusual harm is caused to either the mother or the fetus when the mother is affected (Fig. 2); in some instances the interaction could even be beneficial. ${ }^{19}$ Previously, the only notable exception was found in the accumulation of phenylalanine in a mother with phenylketonuria having a teratogenic effect on a heterozygous fetus. ${ }^{20}$ In LCHAD deficiency and apparently other disorders, a heterozygote woman, carrying an affected fetus, can suffer a life-threatening disease or other complications. The mechanism of the maternal hepatocellular damage is still unclear but is likely to include a fetal accumulation of abnormal fatty acid metabolites that, once passed into the maternal circulation, are capable of overwhelming the mitochondrial oxidation machinery of the heterozygote mother whose metabolism is already challenged by an increased utilization of fatty acids during the late stages of pregnancy. ${ }^{15,16,21}$

The clinical implications arising from the reality that a FATMO disorder may underlie severe maternal complications

Table 2

Disorders of fatty acid transport and mitochondrial oxidation causing maternal complications of pregnancy

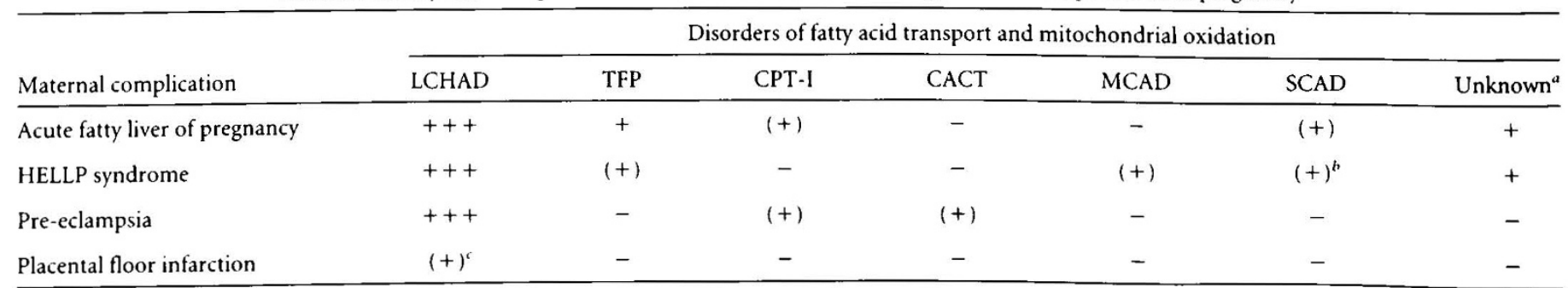

Symbol legend: +++ , association reported in multiple cases; + , association reported in more than one case; $(+)$ association reported in a single case, possibly a coincidental event; - , association not reported. HELLP, hemolysis, elevated liver enzymes, low platelets. For other abbreviations see legend of Table 1 . This table is reproduced with permission, John Wiley \& Sons, Ltd. Rinaldo et al. ${ }^{17}$

"Mothers of children with unspecified disorders but with clinical manifestations and strong biochemical evidence in vivo and in vitro of an underlying FATMO disorder (Rinaldo P et al., unpublished observations).

"Niels Gregersen, personal communication, cited with permission.

'Matern D et al. Placental floor infarction complicating the pregnancy of a fetus with long-chain L-3-hydroxy acyl-CoA dehydrogenase deficiency. Submitted. 


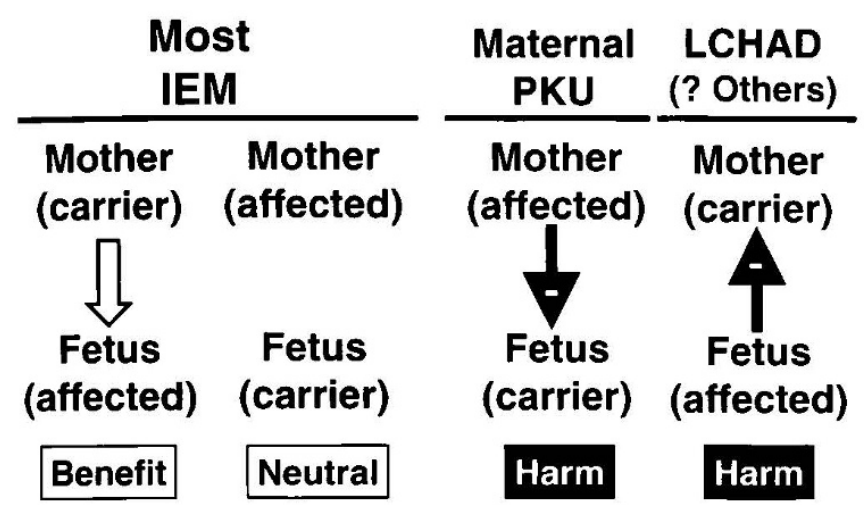

Fig. 2 Inborn errors of metabolism (IEM) and models of maternal-fetal interaction. The open arrow indicates a beneficial effect; the black arrows indicate whether the mother or the fetus has a harmful effect on the other. PKU, phenylketonuria; LCHAD, long-chain L-3-hydroxy acyl-CoA dehydrogenase deficiency.

are multiple and important to recognize. First, the recurrence risk will be $25 \%$, which raises the need of a prenatal diagnosis also to protect the mother's health. Second, all newborns who survive a pregnancy complicated by AFLP should undergo immediate metabolic testing and preventive measures to minimize the risk of life-threatening metabolic decompensation. A molecular genetics approach limited to ruling out the presence of the "common" LCHAD mutation is readily available and relatively inexpensive, but it may not be adequate. Therefore, we recommend following up a negative result with more extensive biochemical testing defined on a case-by-case basis, depending on the clinical course of the newborn. The application of the same approach to all newborns born after pregnancies complicated with HELLP syndrome has been proposed but also criticized, because the finding of an affected newborn is considered to be very low in this scenario. ${ }^{13,21}$

\section{ROLE OF FATMO DISORDERS AS CAUSE OF SUDDEN AND UNEXPECTED DEATH}

Fatty acid oxidation (FAO) disorders might be responsible for up to $5 \%$ of children who die suddenly and unexpectedly. ${ }^{22}$ Pathologists, pediatricians, and geneticists should be challenged to regularly investigate the presence of a possible FATMO disorder and submit for screening all cases with fatty infiltration of the liver and other organs, a family history of sudden death, Reye-like syndrome, or myopathy, and especially a history of lethargy, vomiting, and/or fasting prior to death. ${ }^{12}$ However, caution should be exercised not to use steatosis as the sole criteria to suspect or exclude a possible underlying FAO disorder during the postmortem evaluation of a case of sudden death. In our experience, only 18 of 27 cases diagnosed post mortem with a FATMO disorder showed diffuse microvesicular fatty infiltration of the liver. ${ }^{12}$

Our recommendation is that a frozen specimen of liver and bile and ideally a skin biopsy should be preserved in all cases of sudden death, to be discarded at a later time either if no risk factors are identified or when a credible cause of death has been established. However, a cause of death listed as pneumonia or another acute infectious event should be carefully scrutinized, so that the trigger of an acute event is not confused with the underlying inborn error of metabolism. ${ }^{12}$

Realizing that collection and storage of frozen liver is often problematic, not to mention maintaining a line of cultured fibroblasts, pathologists should be encouraged to collect at least bile, any volume frozen or spotted on filter paper and kept at room temperature, and to retrieve the unused portion of the newborn screening blood spots. Bile acylcarnitine profiling is particularly informative, ${ }^{23}$ likely more reliable than the same analysis of postmortem blood or plasma. The recent availability of mouse knock-out models of FATMO disorders has allowed investigators to perform comparative profiling of plasma and bile specimens. ${ }^{24}$ As shown in Figure 3, simultaneous bile and plasma acylcarnitine profiles obtained after a controlled fasting challenge in the mouse knock-out model of very long-chain acyl-CoA dehydrogenase (VLCAD) deficiency clearly show that biliary excretion represents the predominant route to eliminate toxic acyl-CoA species accumulated in the liver as consequence of FATMO disorders (specimens kindly made available by Dr. Philip A. Wood, University of Alabama).

\section{ROLE OF FATMO DISORDERS AS CAUSE OF ACUTEJ FULMINANT LIVER FAILURE}

Fulminant hepatic failure in children is a clinical syndrome consisting of biochemical evidence of severe hepatic synthetic dysfunction with or without encephalopathy. ${ }^{25}$ Biochemical evidence of severe hepatic dysfunction typically includes prolonged prothrombin time (or specific coagulation factor deficiencies) that does not correct with parenteral vitamin $\mathrm{K}$, hypoglycemia, indirect hyperbilirubinemia and/or hyperammonemia. One of the primary utilities of making a clinical diagnosis of fulminant hepatic failure is the prognostic information that is implied by the diagnosis. Fulminant hepatic failure, which is not the result of acetaminophen overdose, or hepatitis A or B, engenders a predicted mortality of 50 to $80 \%$, including 20 to $30 \%$ who die while on the liver transplant waiting list.

In the vast majority of circumstances, the etiology cannot be discerned. Recently, it has become clear that abnormalities in fatty acid metabolism are associated with fulminant hepatic failure in children, particularly in cases with long-chain fatty acid transport deficiency, ${ }^{26}$ liver-specific short-chain L-3-hydroxy acyl-CoA dehydrogenase deficiency, 27,28 and medium/ short-chain L-3-hydroxy acyl-CoA dehydrogenase deficiency (O'Brien LK et al.: Fulminant hepatic failure associated with mutations in the medium- and short-chain L-3-hydroxyacylCoA dehydrogenase gene, submitted).

Based on these diverse observations, a prospective study has been funded recently by the NIH (Principal Investigator: Dr. Benjamin L. Shneider, Mount Sinai School of Medicine, NY) to investigate a possible underlying FATMO disorder in pediatric cases with acute liver failure who meet the following inclusion criteria: (1) new onset $(<8$ weeks from first symp- 


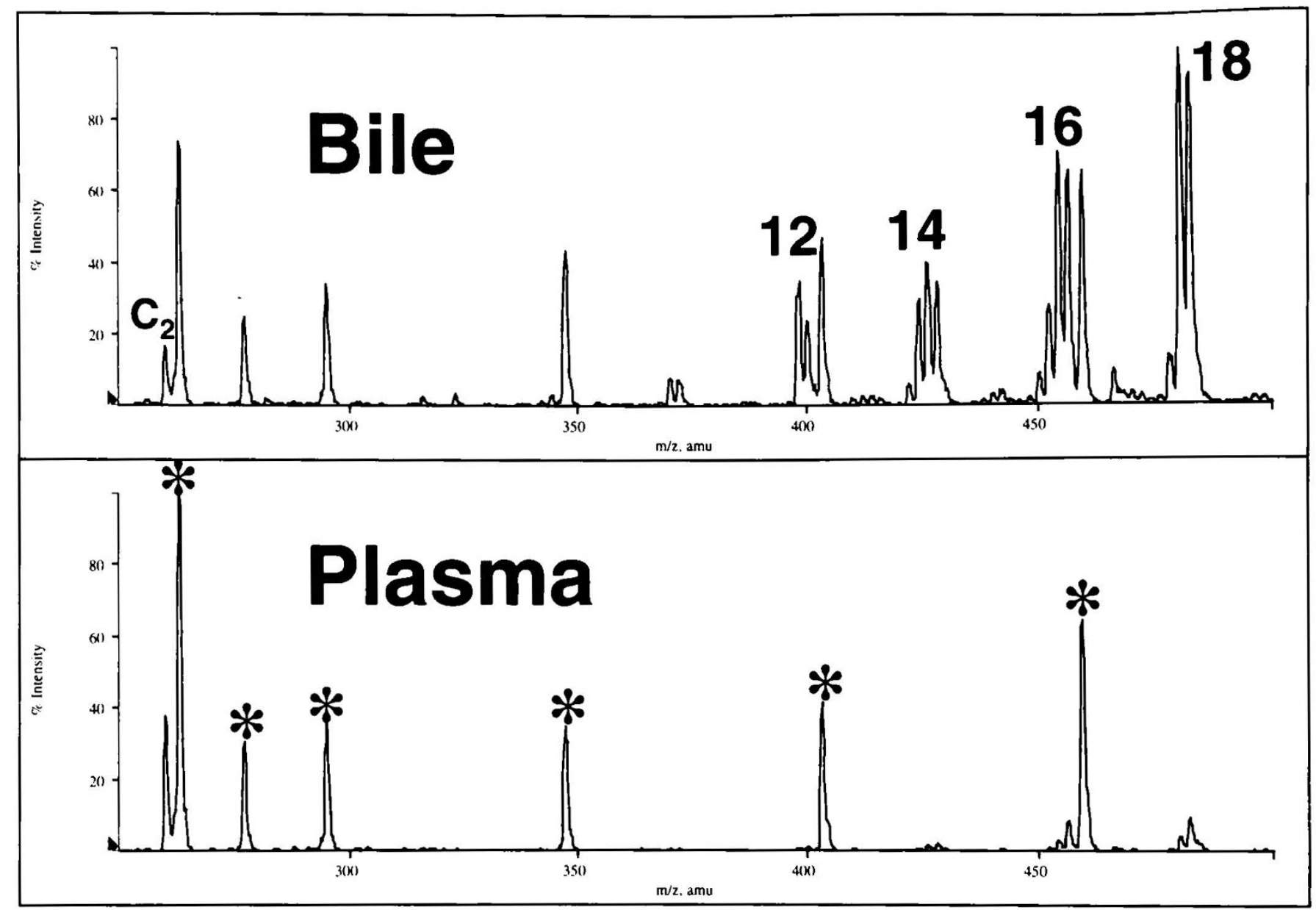

Fig. 3 Butyl-ester acylcarnitine profiles of plasma (top) and bile (bottom) specimens from a very long-chain acyl-CoA dehydrogenase (VLCAD) -/-mouse sacrificed after 24 hours fasting. Internal standards are marked in the lower panel with asterisks. Numeric values $(12.14$, etc.) correspond to the chain length of each cluster, which include saturated, monounsaturated. and diunsaturated acylcarnitine species.

toms), (2) acute hepatic injury with either international normalized ratio (INR) $>1.7$ and encephalopathy or INR $>2.0$, (3) age $<16$ years, (4) informed consent from parent or guardian. Enrollment of cases is expected to start at the beginning of 2001 .

\section{PRENATAL DIAGNOSIS OF FATMO DISORDERS}

To date, all known FATMO disorders are inherited as autosomal recessive traits, with a recurrence risk of $25 \%$. Although prenatal "screening" could occasionally be informative, the pursuit of a prenatal diagnosis after an index case with an unspecified diagnosis should be discouraged, recommending instead aggressive preventive treatment and a thorough evaluation of the newborn immediately after birth. Even when conventional prerequisites to testing have been met, ${ }^{24}$ the prenatal diagnosis of FATMO disorders may still raise both ethical and medical issues. ${ }^{17}$ First, parents face the option of abortion for a treatable disorder like MCAD deficiency or carnitine uptake defect, having experienced the sudden death of one or more children. Obviously, disorders like glutaric acidemia type
2 with congenital anomalies and neonatal CPT II deficiency, characterized by very poor prognosis and outcome, are unquestionable candidates for prenatal diagnosis. Second, the need of prenatal diagnosis could also stem, as mentioned previously, from the need to protect the mother's health. Options and methods available to provide a prenatal diagnosis of FATMO disorders have been discussed in details elsewhere. ${ }^{17}$

\section{PATIENTS WITH UNKNOWN DISORDERS}

The novelty in our understanding of FATMO disorders lies in the concept that severe manifestations could be associated with essentially uninformative biochemical results. Under these circumstances, it is common to encounter patients carrying a tentative diagnosis of being affected with an "unspecified" FATMO disorder. ${ }^{2.4}$ The initial observation may vary from a clinical history of fasting intolerance to a variety of abnormal but nonspecific results of biochemical investigations and in vitro studies. A controlled fasting test, which should be performed only under careful supervision, may have induced hypoglycemia and inadequate ketogenesis, the trademark 
manifestations of FATMO disorders. These patients are treated empirically with low-fat, high-carbohydrate diet, medium-chain triglyceride supplementation, and most importantly strict avoidance of fasting and preventive measures in response to common illnesses. Aggressive treatment of an undefined disorder has caused some confusion among parents and even health professionals, yet new discoveries continue to be made from the bench and bedside study of these atypical cases. ${ }^{26,27,30,31}$ While uninformative results of biochemical investigations and in vitro analysis of cultured fibroblasts could constitute a reasonable end-point of evaluation, they should not be taken as a diagnosis of exclusion, at least not before undertaking a fasting challenge to rule out the presence of a clinically significant fasting intolerance.

In addition to the possibility of yet uncharacterized disorders, an alternative explanation for these unclassified patients could be that they are affected with concurrent partial defects in two or more enzymes, a phenomenon recently described by Vockley et al. as synergistic heterozygosity. ${ }^{32}$ Based on the frequencies of known disorders of energy metabolism, this may represent a relatively common mechanism of disease of potentially great clinical relevance, possibly playing a causative role in combination with susceptibility variants and environmental factors.

For example, short-chain acyl-CoA dehydrogenase (SCAD) deficiency is considered a rare FATMO disorder conventionally diagnosed on the basis of ethylmalonic aciduria and low SCAD activity in cultured fibroblasts. ${ }^{33}$ Gregersen and coworkers have proposed that patients with mild ethylmalonic aciduria may have a "functional" SCAD deficiency due to the presence of either one of two susceptibility SCAD gene variations, i.e., $625 \mathrm{G}>\mathrm{A}$ and $511 \mathrm{C}>\mathrm{T}$, in whom a variable or moderately reduced SCAD activity may be clinically relevant. ${ }^{34,35} \mathrm{~A}$ more recent study has shown that only 1 of 10 newly diagnosed SCAD-deficient patients carried pathogenic mutations in both alleles, while five were double heterozygotes for a pathogenic mutation in one allele and the $625 \mathrm{G}>$ A susceptibility variation in the other. ${ }^{36}$ The remaining four patients carried only either the $511 \mathrm{C}>\mathrm{T}$ or the $625 \mathrm{G}>\mathrm{A}$ variations in each allele. The authors postulated that patients carrying SCAD gene variations could become symptomatic under the influence of additional genetic, cellular, and/or environmental factors.

Clearly, there are a number of issues that remain unsolved concerning the organization and tissue distribution of the components of this pathway. ${ }^{37} \mathrm{With}$ regard to the organization within the mitochondria, it seems logical that the various enzymes should be physically related in a fashion that allows for efficient substrate flow. ${ }^{38}$ To date, however, no such structural organization has been conclusively described for fatty acid oxidation enzymes, but it is possible that some key enzymes and regulatory components involved in this pathway have not yet been described. ${ }^{39-41} \mathrm{~A}$ contribution of conformational abnormalities at the stage of protein folding and cellular handling has also been proposed. ${ }^{42}$ It is tempting to speculate that unclassified cases, synergistic heterozygosity, and common polymorphisms are all connected components of a common scenario, one in which presumed "simple" Mendelian disorders are, in fact, complex traits. ${ }^{42,43}$ The proper recognition of these cases is not impossible, but it will take place only after we learn to look for them properly and creatively in vivo and in vitro.

\section{References}

1. Eaton S, Bartlett K, Pourfarzam M. Mammalian mitochondrial $\beta$-oxidation. Biochem J 1996;320:345-357.

2. Rinaldo P, Raymond $\mathrm{K}, \mathrm{Al}$ Odaib A, Bennett MJ. Fatty acid oxidation disorders: clinical and biochemical features. Curr Opin Pediatr 1998;10:615-621.

3. Saudubray IM, Charpentier C. Clinical phenotypes: diagnosis/algorithms. In: Scriver CR, Beaudet AL, Sly WS, Valle D, editors. The metabolic and molecular bases of inherited disease, 7th ed. New York: McGraw-Hill, 1995:327-400.

4. Bennett MJ, Rinaldo P, Strauss AW. Inborn errors of mitochondrial fatty acid oxidation. Crit Rev Clin Lab Sci 2000;37:1-44.

5. Levy HL. Newborn screening by tandem mass spectrometry: a new era [editorial]. Clin Chem 1998:44:2401-2402.

6. Charrow J, Goodman SI, McCabe ERG, Rinaldo P. Tandem mass spectrometry in newborn screening. Genet Med 2000;2:267-269.

7. Matern D, Rinaldo P. Medium chain acyl-coenzyme A (MCAD) deficiency. In: GeneClinics: medical genetics knowledge base. University of Washington, Seattle. http://www.geneclinics.org/profiles/mcad.

8. Raymond K, Bale AE, Barnes CA, Rinaldo P. Sudden adult death and medium-chain acyl-CoA dehydrogenase deficiency. Genet Med 1999;1:293-294.

9. Wang SS, Fernhoff PM, Hannon H, Khoury MJ. Medium-chain acyl-CoA dehydrogenase deficiency: human genome epidemiology review. Genet Med 1999;1:332339.

10. Matern D, Strauss AW, Hillman SH, Mayatepek E, Millington DS, Trefz F. Diagnosis of TFP deficiency in a blood spot from the newborn screening card by tandem mass spectrometry and DNA analysis. Pediatr Res 1999;46:45-50.

11. Tyni T, Pihko H. Long-chain 3-hydroxyacyl-CoA dehydrogenase deficiency. Acta Paediatr 1999;88:237-245.

12. Boles RG, Buck EA, Blitzer MG, Platt MS, Martin SK, Yoon HR, Madsen JA, ReyesMugica M, Rinaldo P. Retrospective biochemical screening of fatty acid oxidation disorders in postmortem liver of 418 cases of sudden unexpected death in the first year of life. I Pediatr 1998;132:924-933.

13. Den Boer MEJ, Ijlst L, Wijburg FA, Oostheim W, Van Werkhoven MA, Van Pampus MG, Heymans HSA, Wanders RIA. Heterozygosity for the common LCHAD mutation $(1528 \mathrm{G}>\mathrm{C})$ is not a major cause of HELLP syndrome and the prevalence of the mutation in the Dutch population is low. Pediatr Res 2000;48:151-154.

14. Toniges TF, and the AAP Newborn Screening Task Force. Serving the family from birth to the medical home. Newborn screening: a blueprint for the future-a call for a national agenda on state newborn screening programs. Pediatrics 2000;106:389427.

15. Ibdah IA, Bennett MI, Rinaldo P, Zhao Y, Gibson B, Sims H, Strauss AW. A fetal fatty acid oxidation disorder causes maternal liver disease of pregnancy. $N$ Engl J Med 1999;340:1723-1731.

16. Tein I. Metabolic disease in the fetus predisposes to maternal hepatic complications of pregnancy [editorial]. Pediatr Res 2000;47:6-8.

17. Rinaldo P, Studinski A, Matern D. Prenatal diagnosis of disorders of fatty acid transport and mitochondrial oxidation [invited commentary]. Prenat Diagnosis. In press.

17a. Matern D, Hart P, Murtha AP, Vockley I. Gregersen N, Millington DS, Treem WR. Acute fatty liver of pregnancy associated with short-chain acyl-coenzyme A dehydrogenase deficiency. J Pediatr. In press.

18. Nelson J, Lewis B, Walters B. The HELLP syndrome associated with fetal mediumchain acyl-CoA dehydrogenase deficiency. I Inherit Metab Dis 2000;23:518-519.

19. Wasserstein MP, Gaddipati S, Snyderman SE, Eddleman K, Desnick RJ, Sansaricq C. Successful pregnancy in severe methylmalonic acidaemia. J Inherit Metab Dis 1999; 22:788-794.

20. Platt LD, Koch R, Hanley WB, Levy HL, Matalon R, Rouse B, Trefz F, de la Cruz F, Guttler F, Azen C. Friedman EG. The international study of pregnancy outcome in women with maternal phenylketonuria: report of a 12-year study. Am J Obstet Gynecol 2000;182:326-333.

21. Strauss AW, Bennett MI, Rinaldo P, Sims HF, O'Brien LK, Zhao Y, Gibson B, Ibdah I. Inherited long-chain 3-hydroxyacyl-CoA dehydrogenase deficiency and a fetalmaternal interaction cause maternal liver disease and other pregnancy complications. Semin Perinatol 1999;23:100-112.

22. Rinaldo P, Yoon HR, Yu C, Raymond K, Tiozzo C, Giordano G. Sudden and unexpected neonatal death: a protocol for the postmortem diagnosis of fatty acid oxidation disorders. Semin Perinatol 1999;23:204-210. 
23. Rashed MS, Ozand PT, Bennett MJ, Barnard JJ, Govindaraju DR, Rinaldo P. Diagnosis of inborn errors of metabolism in sudden death cases by acylcarnitine analysis of postmortem bile. Clin Chem 1995;41:1109-1114.

24. Kurtz D, Rinaldo P, Rhead WJ, Tian L, Millington DS, Vockley G, Hamm D, Brix AE, Lindsey IR, Pinkert CA, O'Brien WE, Farmer SC, Wood PA. Targeted disruption of mouse long-chain acyl-CoA dehydrogenase reveals crucial role in fatty acid oxidation. Proc Natl Acad Sci USA 1998;95:15592-15597.

25. Bhaduri B, Mieli-Vergani G. Fulminant hepatic failure: pediatric aspects. Semin Liver Dis 1996;116:349-355.

26. Al Odaib A, Shneider BL, Bennett MJ, Pober BR, Reyes-Mugica M, Friedman AL, Suchy FI, Rinaldo P. A defect in the transport of long-chain fatty acids associated with acute liver failure. N Engl J Med 1998:339:1752-1757.

27. Bennett MI, Spotswood SD, Ross KF, Comfort S, Koonce R, Boriack RL, IJlst L, Wanders RI. Fatal hepatic short-chain L-3-hydroxyacyl-coenzyme A dehydrogenase deficiency: clinical, biochemical, and pathological studies on three subjects with this recently identified disorder of mitochondrial beta-oxidation. Pediatr Dev Pathol 1999;2:337-345.

28. Treacy EP, Lambert DM, Barnes R, Boriack RL, Vockley J, O'Brien LK, Jones PM, Bennett MI. Short-chain hydroxyacyl-coenzyme A dehydrogenase deficiency presenting as unexpected infant death: a family study. J Pediatr 2000;137:257-259.

29. Rinaldo P, Matern D. Biochemical diagnosis of inborn errors of metabolism. In: Rudolph CD, Rudolph AM, Hostetter M, Lister G, Siegel N, editors. Rudolph's pediatrics, 21st ed. New York: McGraw-Hill. In press.

30. Kamijo T, Indo I, Souri M, Aoyama T, Hara T, Yamamoto S, Ushikubo S, Rinaldo P, Matsuda I, Komiyama A, Hashimoto T. Medium-chain 3-ketoacyl-CoA thiolase deficiency: a new disorder of mitochondrial fatty acid $\beta$-oxidation. Pediatr Res 1997; 42:569-576.

31. Thompson GN, Hsu BYL, Pitt JJ, Treacy E, Stanley CA. Fasting hypoketotic coma in a child with deficiency of mitochondrial 3-hydroxy-3-methylglutaryl-CoA synthase. N Engl J Med 1997;337:1203-1207.

32. Vockley J, Rinaldo P, Bennett MJ, Matern D, Vladutiu GD. Synergistic heterozygosity: metabolic disease resulting from multiple partial defects in one or more metabolic pathways. Mol Genet Metab 2000;71:10-18.

33. Bhala A, Willi SM, Rinaldo P, Bennett MJ, Schmidt-Sommerfeld E, Hale DE. Clinical and biochemical characterization of short-chain acyl-coenzyme A dehydrogenase deficiency. J Pediatr 1995;126:910-915.
34. Corydon MJ, Gregersen N, Lehnert W, Ribes A, Rinaldo P, Kmoch S, Christensen E, Kristensen TJ, Andresen BS, Bross P, Winter V, Madsen IM, Martinez G, Neve S, Kristiansen K, Jensen TG, Bolund L, Kølvraa S. Ethylmalonic aciduria is associated with an amino acid variant (G209S) of short-chain acyl-coenzyme A dehydrogenase (SCAD). Pediatr Res 1996;39:1059-1066.

35. Gregersen N, Winter V, Corydon MJ, Corydon TJ, Rinaldo P, Ribes A, Martinez G, Bennett MJ, Vianey-Saban C, Bhala A, Hale DE, Lehnert W, Kmoch S, Roig M, Riudor E, Einberg H, Andresen BS, Bross P, Bolund LA, Kølvraa S. Identification of four new mutations in the short-chain acyl-CoA dehydrogenase (SCAD) gene in two patients: one of the variant alleles, $511 \mathrm{C}>\mathrm{T}$, is present at an unexpectedly high frequency in the general population, as was the case for $625 \mathrm{G}>\mathrm{A}$, together conferring susceptibility to ethylmalonic aciduria. Hum Mol Genet 1998;7:619-627.

36. Corydon MJ, Vockley G, Rinaldo P, Rhead WJ, Kjeldsen M, Winter V, Riggs C, Babovic-Vuksanovic D, Smeitink J, de Jong J, Roe CR, Levy HL, Sewell AC, Matern $D$, Dasouki M, Gregersen N. Role of common variant alleles in the molecular basis of short-chain acyl-CoA dehydrogenase deficiency. Pediatr Res. In press.

37. Eaton $S, B a r t l e t t K$. Tissue specific differences in intramitochondrial control of $\beta$-oxidation. In: Quant PA, Eaton S, editors. Current views of fatty acid oxidation and ketogenesis: from organelles to point mutations. New York: Kluwer, 1999:161-168.

38. Parker A, Engel PC. Preliminary evidence for the existence of specific functional assemblies between enzymes of the beta-oxidation pathway and the respiratory chain. Biochem J 2000;345:429-435.

39. Leone TC, Weinheimer CJ, Kelly DP. A critical role for the peroxisome proliferatoractivated receptor alpha (PPAR $\alpha$ ) in the cellular fasting response: the PPAR $\alpha$-null mouse as a model of fatty acid oxidation disorders. Proc Natl Acad Sci USA 1999;96: 7473-7478.

40. Telford EAR, Moynihan LM, Markham AF, Lench NJ. Isolation and characterization of a cDNA encoding the precursor for a novel member of the acyl-CoA dehydrogenase gene family. Biochim Biophys Acta 1999;1446:371-376.

41. Boss $\mathrm{O}$, Hagen T, Lowell BB. Uncoupling proteins 2 and 3: potential regulators of mitochondrial energy metabolism. Diabetes 2000;49:143-156.

42. Gregersen N, Bross P, Jørgensen MM, Corydon TJ, Andresen BS. Defective folding and rapid degradation of mutant proteins is a common disease mechanism in genetic disorders. J Inherit Metab Dis 2000;23:441-447.

43. Dipple KM, McCabe ERB. Phenotypes of patients with "simple" Mendelian disorders are complex traits: thresholds, modifiers, and system dynamics. Am J Hum Genet 2000;66:1729-1735. 